Artículo

\title{
Impacto de las actividades agropecuarias y petroleras sobre las coberturas naturales del campo petrolero Samaria, Tabasco
}

Rodimiro Ramos-Reyes ${ }^{1}$

Miguel Á. Palomeque-De la Cruz ${ }^{2}$

Joel Zavala Cruz ${ }^{3 \S}$

${ }^{1}$ El Colegio de la Frontera Sur-Unidad Villahermosa. Carretera a Reforma km 15.5, s/n, Ra. Guineo $2^{\text {da. }}$ Sección Centro, Tabasco, México. CP. 86280. (rramos@ecosur.mx). ${ }^{2}$ Universidad Juárez Autónoma de Tabasco-División Académica de Ciencias Biológicas. Carretera Villahermosa-Cárdenas km 0.5, s/n, entronque a Bosques de Saloya, Villahermosa, Tabasco. CP. 86150. (migueldacbiol@ hotmail.com). ${ }^{3}$ Colegio de Postgraduados-Campus Tabasco. Periférico Carlos A. Molina s/n, H. Cárdenas, Tabasco. CP. 86500. Tel. 9373722386.

${ }^{\S}$ Autor para correspondencia: zavala_cruz@colpos.mx.

\section{Resumen}

En las regiones tropicales del sureste de México, las actividades productivas de subsistencia, el crecimiento de las ciudades y la industria petrolera, están ocupando coberturas originales de vegetación selvática, vegetación hidrófita y cuerpos de agua. El objetivo del estudio fue valuar el efecto provocado por el crecimiento de las actividades primarias y de extracción de petróleo sobre las coberturas naturales, mediante la modelación del cambio de uso del suelo en el campo petrolero Samaria en los municipios de Cunduacán y Centro, Tabasco, México. Se realizó un análisis espacial con mapas de ocupación del suelo mediante Land Change Modeler y se determinaron ganancias, pérdidas, contribuciones, el cambio neto y las transiciones de cada categoría. Desde el inicio de la extracción de petróleo en 1965 hasta 2019, en el CPS, con una superficie de 8052 ha, se perdieron 647 ha de humedales y 436 ha de vegetación arbórea, como consecuencia de la expansión de actividades primarias y la industria petrolera, las cuales ocuparon 1287 ha de uso agrícola, 1598 ha de uso pecuario, 269 ha de infraestructura petrolera y 775 ha de la zona urbana. Se concluye que la aplicación de Land Change Modeler fue novedosa para la evaluación de la degradación de los ecosistemas y la estimación de la distribución del cambio de coberturas naturales y usos artificiales en el campo petrolero Santamaria, estado de Tabasco.

Palabras clave: actividades agropecuarias, cambio de uso del suelo, petróleos mexicanos.

Recibido: agosto de 2021

Aceptado: octubre de 2021 


\section{Introducción}

Debido a la creciente demanda por bienes como alimento, vivienda, agua potable y otros servicios, las actividades antropogénicas generan constante presión sobre los recursos naturales en amplias escalas geográficas, lo que afecta su estructura, funcionamiento y distribución (Vitousek et al., 1997; Challenger y Dirzo, 2009). Estas actividades provocan cambio de uso del suelo que transforma la cubierta vegetal original a otros usos y degrada la calidad de la vegetación, lo que modifica la densidad y composición de las especies de plantas. Entre las consecuencias más importantes del cambio de uso de suelo se encuentra la pérdida de la biodiversidad y los servicios ambientales (Balvanera, 2012).

El cambio de uso del suelo, en su sentido más amplio, se refiere a las diferentes formas en que se emplea un terreno y su cobertura vegetal o acuática (Trucíos-Caciano et al., 2013). El cambio de uso como consecuencia de las actividades agropecuarias induce pérdida de biodiversidad, al fragmentar y destruir los diferentes hábitats, disminuye la capacidad de purificación del agua, la regeneración de los suelos, el reciclaje de nutrientes (Zavala-Cruz y Castillo-Acosta, 2007) y genera emisiones de gases de efecto invernadero que representan un cuarto de las emisiones globales actuales (Tubiello et al., 2015).

La conversión de bosques tropicales a superficies agrícolas y pecuarias destaca entre los mayores orígenes de la deforestación y degradación ambiental (Davidson et al., 2012). En México, además del uso agropecuario, el crecimiento urbano ha provocado depredación de los recursos naturales (Sánchez y Batres, 2006), la pérdida de bosques tropicales y templados, vegetación desértica y humedales (Torres-Vera et al., 2009; Zepeda-Gómez et al., 2012), con una tasa de cambio anual negativa $(-0.08 \%$ - $0.41 \%)$ para bosques, selvas, matorrales y mezquitales (Rosete-Vergés et al., 2014).

Estos indicadores son consistentes con la tasa de deforestación de $0.3 \%$ de los bosques templados y tropicales entre 1990-2015 en México (FAO, 2015). Los humedales enfrentan deterioro ambiental debido a la desecación para la expansión de áreas urbanas, agrícolas, acuícolas, pecuarias (Brena, 2016) y el incremento de la infraestructura de extracción de petróleo (Tudela, 1992; Tubiello et al., 2015). Uno de los mayores impactos del cambio de uso del suelo ha sido la degradación de 45\% de los suelos de México (Ortiz-Solorio et al., 2011). En el Sureste de México, Tabasco se convirtió en el mayor productor de hidrocarburos del país en la década de los años setenta (Capdepont-Ballina y Marín-Olán, 2014), lo que generó un impulso social y económico que repercutió en el crecimiento de las ciudades, mayormente Villahermosa (Bazant, 2010).

En 1978 el PIB estatal provenía tres cuartas partes de la producción de hidrocarburos, pero el sector agropecuario decreció más de la mitad (Lezama, 1987). El auge petrolero aceleró el crecimiento de las zonas urbanas y magnificó el deterioro ambiental de los ecosistemas adyacentes al basarse en rellenos de lagunas y zonas de inundación (Sánchez-Munguía, 2005). En este contexto se ubica el CPS que produce, junto con el campo Luna, 127 mil barriles de petróleo día ${ }^{-1}$, siendo el más productivo en la zona terrestre de México (PEMEX, 2016). 
El análisis de los cambios en territorios urbanizados, rurales y naturales requiere la modelación del cambio de uso del suelo para predecir escenarios ambientales y socioeconómicos y establecer políticas de planificación territorial considerando la interacción de los factores socioeconómicos y biofísicos en un espacio geográfico (Paegelow et al., 2003; Pineda-Jaimes et al., 2009). La aplicación de uno o varios modelos de cambio de uso del suelo permite comprender sus alcances, los factores causales y las consecuencias, esta información contribuye a generar propuestas de ordenamiento territorial (Jiménez-Moreno et al., 2011).

En ese sentido, Land Change Modeler (LCM) es un sistema innovador que da soporte a la toma de decisiones de planificación de la tierra, con un flujo de trabajo automatizado y fácil de usar, simplifica las complejidades del análisis de cambios, permite analizar rápidamente el cambio de cobertura de la tierra, modelar empíricamente las relaciones con las variables explicativas y simular futuros escenarios de cambio de la tierra (Eastman, 2012).

Dicho sistema está orientado a evaluar problemas de conversión acelerada del terreno y contribuye a resolver las necesidades analíticas de la conservación de la biodiversidad, además integra el análisis de los datos, la noción de cambios de usos del suelo y la predicción de escenarios (Camacho-Olmedo et al., 2010; Eastman, 2012). El objetivo del estudio fue analizar el cambio de uso del suelo sobre ecosistemas de humedales y vegetación arbórea por efecto de actividades petroleras, agropecuarias y urbanas en el CPS, estado de Tabasco.

\section{Materiales y métodos}

\section{Área de estudio}

El campo petrolero Samaria (CPS) se ubica en el centro-sur de Tabasco, en una superficie de 8 052 ha, en los municipios de Cunduacán y de Centro (Figura 1). Tiene clima cálido húmedo con abundantes lluvias en verano, temperatura media anual de $25{ }^{\circ} \mathrm{C}$ y precipitación anual de $2000 \mathrm{~mm}$. Forma parte de la región fisiográfica Planicie Costera del Golfo y el paisaje geomorfológico de planicie fluvial, los ríos Samaria al norte y Carrizal al sur, han modelado diques naturales, llanuras de inundación y cubetas de decantación, sobre sedimentos aluviales del Cuaternario Holoceno, con alturas de 4 a $10 \mathrm{msnm}$. Prevalecen usos de pastizal, cultivos de plátano, cacao y maíz, vegetación secundaria, infraestructura petrolera y asentamientos humanos (Zavala-Cruz et al., 2003).

\section{Preparación de la base de datos geográfico}

Se utilizaron fotografías aéreas de 1965 escala 1:30 000 del INEGI y una imagen satelital Sentinel2 del año 2019 a una resolución de $10 \mathrm{~m}$, proporcionado de la agencia espacial europea de la plataforma (https://scihub.copernicus.eu/dhus/\#/home). Se utilizaron estos dos períodos porque 1965 es cuando inicio la actividad petrolera en el campo Samaría y 2019 por ser algo más actual. Los usos del suelo y las coberturas naturales se identificaron mediante fotointerpretación y digitalización de polígonos vectoriales a escala de observación en pantalla de 1:15 000, aplicando los criterios de tono, forma, tamaño y textura (Ramos-Reyes et al., 2016). 

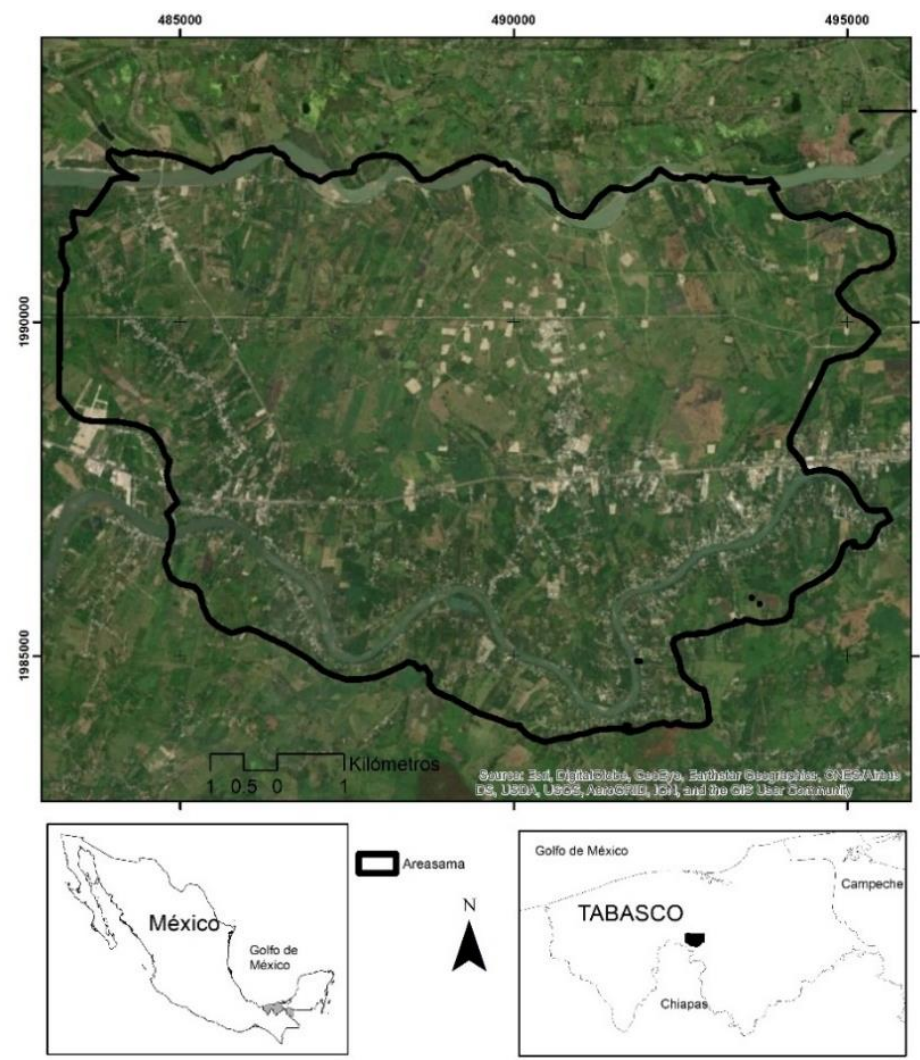

Figura 1. Localización del campo petrolero Samaria, Tabasco.

Se empleó un Datum WGS84-Proyección UTM zona 15N y el proceso de digitalización se realizó con el programa Arc GIS 10.5 ${ }^{\circledR}$. La digitalización del vector de 1965 fue corroborada con información histórica del campo Samaria (Zavala-Cruz et al., 2003) y la del vector de 2019 fue apoyada con una supervisión en campo y comparación con fuentes cartográficas de otros estudios realizados en Samaria y estudios sobre suelos de Tabasco (Zavala-Cruz et al., 2003; Zavala-Cruz et al., 2007) y ubicación de sitios con equipo GPS.

Es primordial señalar que para asegurar que fuera equivalente el cruce de las categorías de uso del suelo y coberturas con las imágenes en Land Change Modeler y el cruce de tablas con CrossTab, se decidió unificar la metodología de clasificación con una reclasificación con el comando Reclassify de Arc GIS $10.5^{\circledR}$, obteniendo: 1) humedales (ríos, lagunas y pantanos); 2) arbórea (selva mediana y vegetación riparía); 3) agrícola (cultivos); 4) pecuario (pastizales con diferentes niveles de manejo y en asociación con matorrales y zarzales sujetos a inundación temporal); y 5) infraestructura petrolera.

Los vectores reclasificados (1965 y 2019) fueron transformados a formato raster con un tamaño de pixel de 20 m incluyendo 370 columnas y 252 renglones con el comando Polygon to Raster de ArcToolbox, dentro del programa Arc Gis $10.5^{\circledR}$, esta reducción de pixeles facilita que los modelos de análisis espacial programa IDRISI TerrSet ${ }^{\circledR}$ puedan ejecutarse con mayor rapidez sin afectar los valores espaciales obtenidos en la digitalización de vectores en Arc Gis. Posteriormente los dos archivos raster (1965 y 2019) fueron exportados a formato TIF para facilitar la exportación de los dos archivos al programa IDRISI TerrSet ${ }^{\circledR}$ empleando el comando GeoTIFF/Tiff to Idrisi y posteriormente ser utilizados para el cruce de imágenes con Land Change Modeler y también para el cruce de tablas con CrossTab. 


\section{Modelación del cambio de uso del suelo 1965-2019 (LCM)}

El análisis del cambio de uso del suelo se llevó a cabo con Land Change Modeler (LCM) de IDRISI Selva ${ }^{\circledR}$. Este módulo se fundamenta en el análisis de dos fechas mediante una matriz de tabulación cruzada que consiste en una tabla con arreglos simétricos (Comando CrossTab) (Cuadro 1), el cual permite encontrar para cada categoría de uso del suelo y coberturas, las ganancias y las pérdidas, el cambio neto y contribuciones entre categorías experimentado entre dos momentos temporales (Cuadro 1).

Cuadro 1. Matriz de tabulación cruzada (CrossTab) para dos mapas de diferentes fechas (Pontius et al. (2004).

\begin{tabular}{|c|c|c|c|c|c|c|c|c|}
\hline & Tiempo 2 & & & & & & & \\
\hline & 1 & 2 & 3 & 4 & 5 & 6 & 7 & \\
\hline 1 & Tiempo 1 & & Clase 1 & Clase 2 & & Cla & suma T 1 & Pérdidas \\
\hline 2 & Clase 1 P11 & & P12 & & $\mathrm{P} 1 \mathrm{n}$ & P $1+$ & $\mathrm{P} 1+-\mathrm{pjj}$ & \\
\hline 3 & Clase 2 P21 & & P12 & & $\mathrm{P} 2 \mathrm{n}$ & P 2+ & $P 2+-p j j$ & \\
\hline 4 & & & & & & & & \\
\hline 5 & Clase n P n1 & & $\operatorname{Pn} 2$ & & Pnn & $\mathrm{Pn}+$ & Pn+ - pjj & \\
\hline 6 & Total T2 $\mathrm{P}+1$ & & $\mathrm{P}+2$ & & $\mathrm{P}+\mathrm{n}$ & $\mathrm{P}$ & $\mathrm{P}$ & \\
\hline 7 & Ganancias & & $\mathrm{P}+1$ & $\mathrm{Pjj} \quad \mathrm{P}+2$ & & $P+n$ & $\mathrm{Pjj}$ & \\
\hline
\end{tabular}

Asimismo, facilita valorar el cambio total tomando como referencia las persistencias y así evaluar cuáles fueron las transiciones de un estado de la superficie terrestre a otro (Pineda-Jaimes et al., 2009; Pontius et al., 2012). En dicha matriz las filas representan las categorías del mapa en el tiempo 1 (T1) y las columnas las categorías del mapa en el tiempo 2 (T2). Asimismo, la diagonal principal muestra las persistencias entre el $\mathrm{T} 1$ y T2, mientras que los elementos fuera de la diagonal principal dan cuenta de las transiciones ocurridas entre el T1 y T2 para cada categoría.

En la fila 6 se recoge el total ocupado por cada categoría en el T2 $(\mathrm{P}+\mathrm{j})$, mientras que en la columna 6 se recoge el total de cada categoría en el tiempo $\mathrm{T} 1$ (Pi+). La fila 7 muestra la ganancia que tuvo cada categoría entre el T1 y T2 y la columna 7 la pérdida que tuvo cada categoría entre el T1 y T2 (Pontius et al., 2004; Pineda-Jaimes et al., 2009). Una vez obtenida la matriz de tabulación cruzada, se calcularon los siguientes parámetros para el análisis de los cambios de ocupación del suelo (Pontius et al., 2004; Pineda-Jaimes et al., 2009): las ganancias, expresadas como la diferencia de la suma total de la fila 6 y los valores de la diagonal principal; es decir, Gij = P+j - Pjj; las pérdidas, expresadas como la diferencia de la suma total de la columna 6 y los valores de la diagonal principal; es decir, $\mathrm{Lij}=\mathrm{Pj}+-\mathrm{Pjj}$.

El cambio neto, expresado como el valor absoluto de la diferencia de las pérdidas y las ganancias de cada categoría $\mathrm{Dj}=\mid \mathrm{Lij}$ - Gij|; las transiciones, expresadas como el doble del valor mínimo de las ganancias o las pérdidas; es decir, $\mathrm{Sj}=2 \times \mathrm{MIN}(\mathrm{Pj}+-\mathrm{Pjj}, \mathrm{Pj}+-\mathrm{Pjj})$. Con el módulo land change modeler (LCM) se generó la matriz de tabulación cruzada (CrossTab) entre los mapas de uso del suelo y coberturas de 1965 y 2019 (Figura 2). Las imágenes de 1965 y 2019 fueron cruzados con el módulo LCM y Crosstab para obtener una matriz de probabilidades de cambio validada. 
El módulo CrosstaB generó las probabilidades de, Cramer's V = 0.99, y Overall Kappa: 0.99 (Cuadro 2), demostrando que ambas imágenes representan un análisis espaciotemporal preciso y apegado a la realidad del territorio. Los resultados (1965-2019) incluyen un resumen cartográfico, tabular y gráfico que muestra la superficie de cada categoría, en comparación con otras, en términos de persistencias, ganancias, pérdidas, contribuciones y transiciones.

Cuadro 2. Matriz de tabulación cruzada en pixeles (CrossTab) 1965 vs 2019.

\begin{tabular}{ccccccc}
\hline Categoría & Humedales & Arbórea & Agrícola & Pecuario & Infraestructura petrolera & Total \\
\hline Humedales & 7728 & 1264 & 349 & 2703 & 13 & 12057 \\
Arbórea & 599 & 970 & 1691 & 1623 & 0 & 4883 \\
Agrícola & 1671 & 2375 & 8850 & 6714 & 1 & 19611 \\
Pecuario & 6219 & 3430 & 3618 & 8240 & 25 & 21532 \\
Infraestructura petrolera & 879 & 194 & 263 & 913 & 53 & 2302 \\
Urbano & 369 & 294 & 4154 & 1663 & 2 & 6482 \\
Total & 17484 & 8532 & 18943 & 21899 & 94 & 93240 \\
\hline
\end{tabular}

Chi-square $=465472.2188 ; \mathrm{df}=25 ; p$-Level= 0; Cramer's V= 0.9992; Overall Kappa= 0.9988. Nota: renglones 2019, columnas 1964.

\section{Tasa de cambio de uso del suelo}

La tasa de cambio de uso del suelo identifica el tipo y magnitud de la presión sobre los recursos naturales e indica de manera porcentual el cambio anual de la superficie de una categoría de uso al inicio de cada año de análisis (Mas et al., 2002; Palacio-Prieto et al., 2004). Se utilizaron los mapas de uso del suelo y coberturas de 1965 y 2019 (Figura 2) y para su cálculo se empleó la fórmula d $=\left[(\mathrm{S} 2 / \mathrm{S} 1)^{1 / \mathrm{n}}-1\right] 100$. Donde: $S 1$, es el área cubierta por determinado uso del suelo al inicio del período; $S 2$, es el área cubierta por determinado uso del suelo al final del periodo y $\mathrm{T}$, es el número de años del periodo de análisis (Palacio-Prieto et al., 2004).
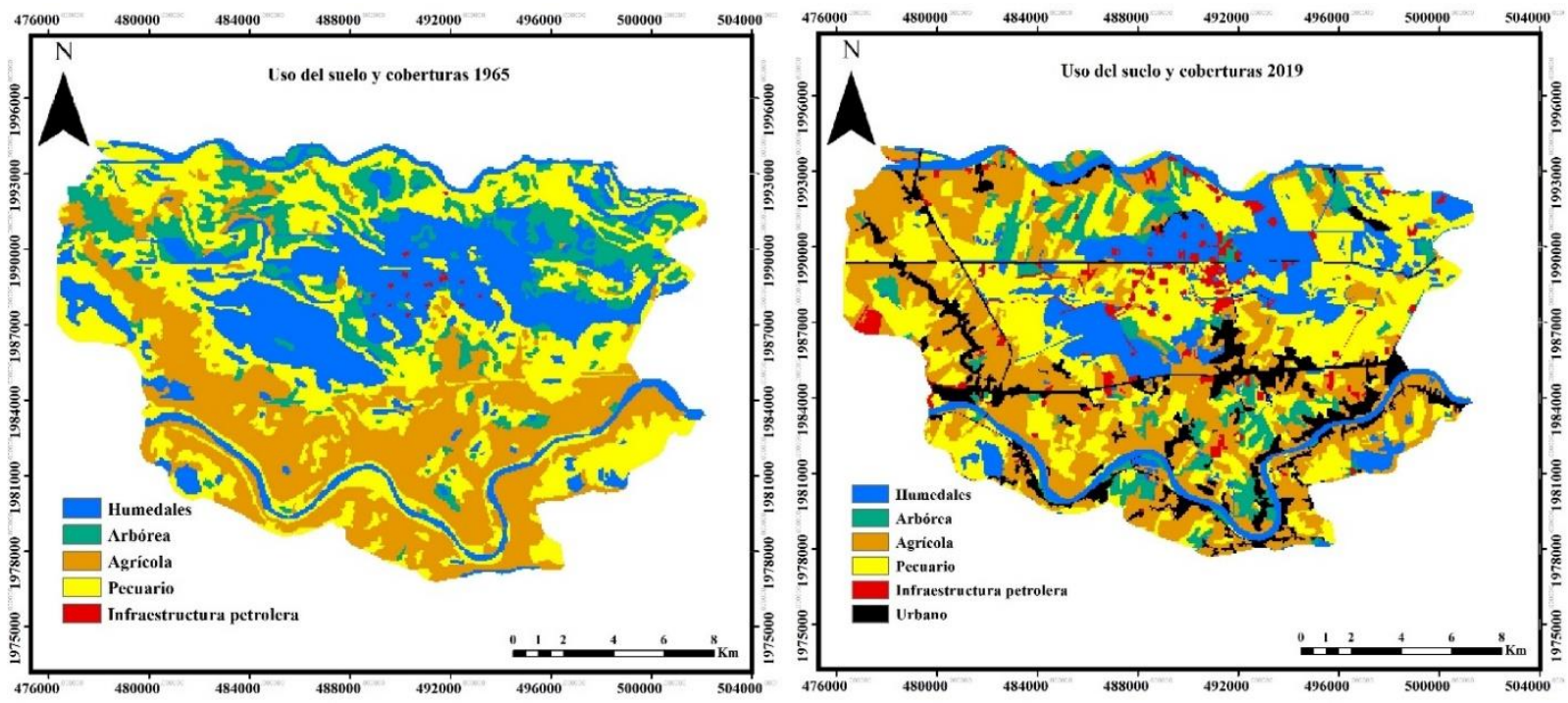

Figura 2. Uso del suelo y coberturas en el campo petrolero Samaria, Tabasco (1965 y 2019). 


\section{Resultados y discusión}

\section{Cambio de uso del suelo (1965-2019)}

En 1965 el 26.1\% del área del CPS correspondía a humedales, y el 12\% estaba representada por vegetación arbórea; la zona agrícola y pecuaria ocupaban las mayores superficies (28.3 y 32.7\%, respectivamente), y la infraestructura para la extracción de petrolero apenas representaba $0.1 \%$ del territorio (Figura 2 y Cuadro 2). En 2019 se detectó que las coberturas de humedales y vegetación arbórea pasaron a ocupar $18 \%$ y $7.3 \%$, las áreas agrícolas y pecuarias mantuvieron la mayor superficie (29.3 y 32.2\%), la infraestructura petrolera incrementó su área (3.4\%) y apareció el uso urbano (9.7\%) (Figura 2 y Cuadro 2). Cabe resaltar, que en el cálculo de las pérdidas y ganancias (Cuadro 2) y el mapa de pérdidas, persistencias y ganancias para cada categoría (Figura 3).

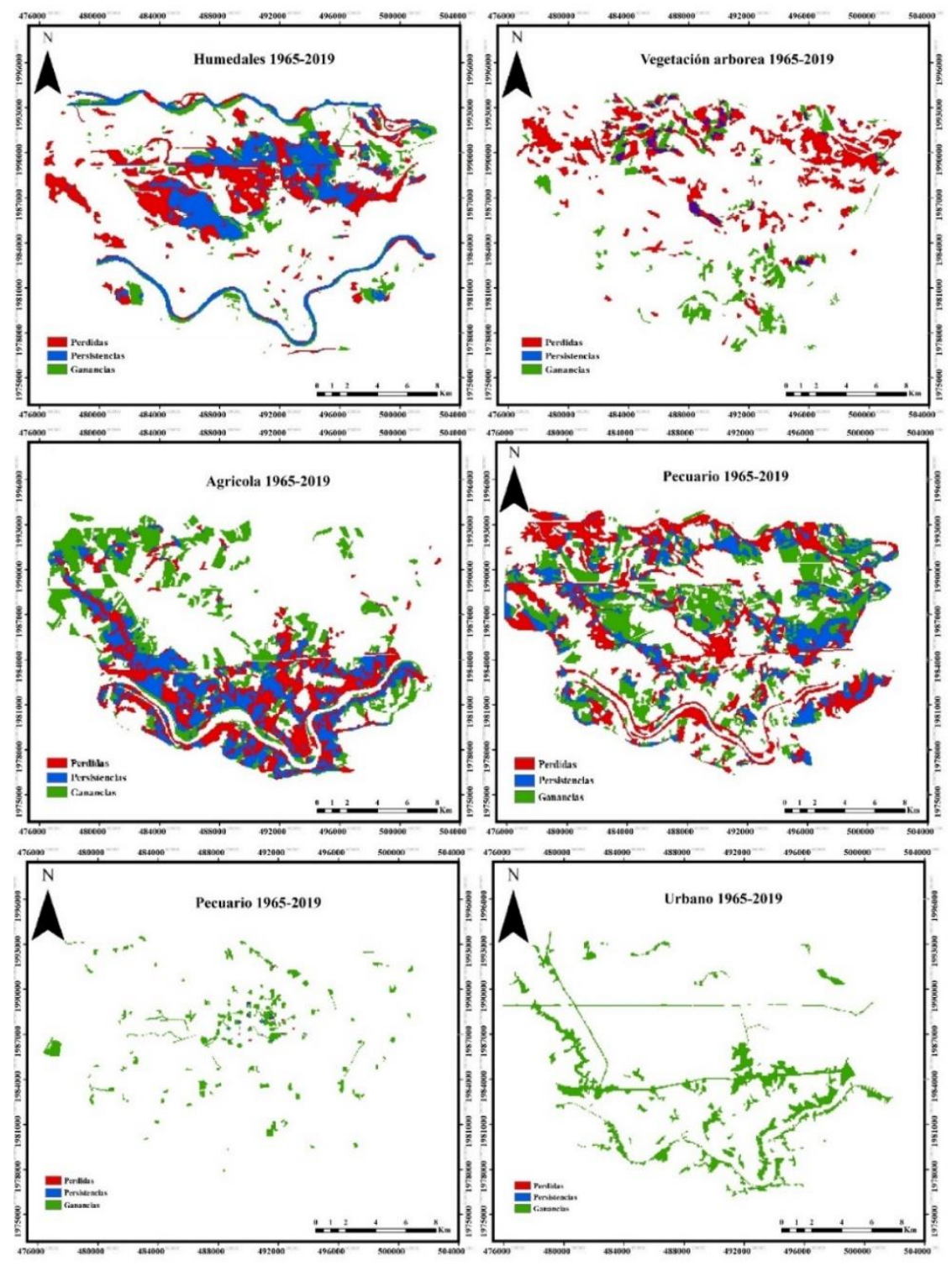

Figura 3. Mapas de pérdidas, persistencias y ganancias en el campo petrolero Samaria, Tabasco (1965-2019). Humedales y vegetación arbórea. 
El análisis de las contribuciones entre las categorías muestra que la pérdida de coberturas de humedales durante las cinco décadas se relacionó con el aumento de 158 ha de área agrícola, 480 ha de superficie pecuaria y 104 ha de infraestructura petrolera (Figura 4). La degradación de esta vegetación coincide con la disminución de humedales en el entorno de Villahermosa que se relacionó con la modificación de su interconexión hidráulica mediante la construcción de canales, infraestructura urbana y caminos, así como la regulación de cauces de ríos para retención o desviación del agua, el dragado de humedales para control de inundaciones y la extracción de agua para usos agropecuarios (Hettiarachchi et al., 2014; Sánchez et al., 2015).

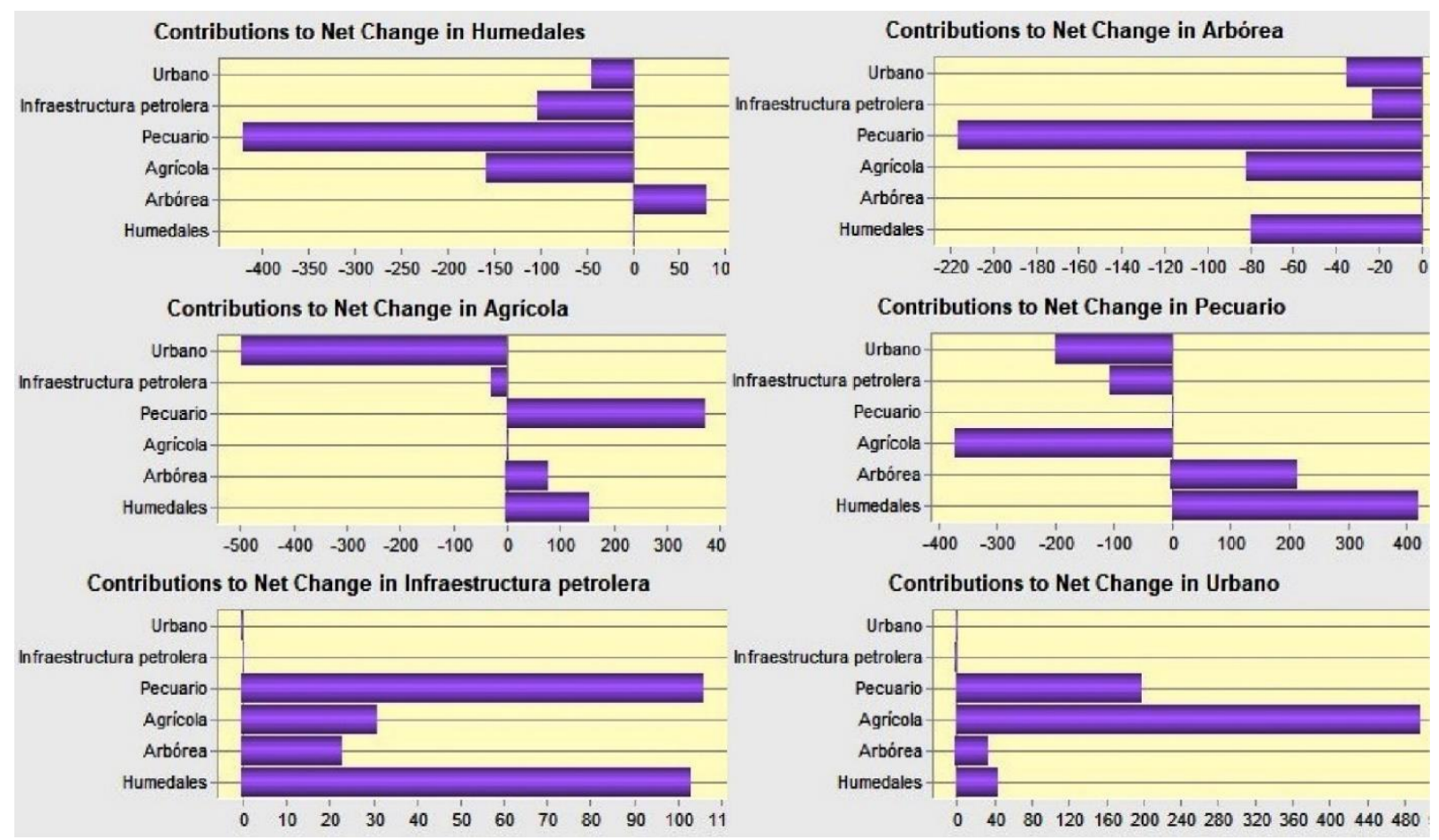

Figura 4. Contribuciones de superficies (ha) para las coberturas naturales en el campo petrolero Samaria, Tabasco (1965-2019).

En otros humedales como en la Reserva de la Biosfera Pantanos de Centla, ubicado en el delta de los ríos Usumacinta y Grijalva, la perdida de humedales también se ha atribuido a la apertura de vías de comunicación a actividades de la industria petrolera, la sobreexplotación de recursos naturales y la expansión ganadera y agrícola (Guerra-Martínez y Ochoa-Gaona, 2006). De la misma forma, las pérdidas de vegetación arbórea en el CPS fueron causados por el establecimiento de 216 ha del uso pecuario y 82 ha de uso agrícola (Figura 4).

La tasa de cambio de la vegetación arbórea registrada en el CPS fue del 1\%, siendo inferior a la de Villahermosa que se ubicó en $4.63 \%$ en el periodo 1984-2008, debido a que perdió 4008 ha, causando uno de los mayores impactos en las últimas tres décadas en Tabasco (Palomeque-De la Cruz et al., 2017a). Las tasas de pérdida de vegetación arbórea y humedales conllevan la desaparición de servicios ambientales como la provisión de agua y alimento, el mantenimiento de la biodiversidad de flora y fauna y la regulación del clima e inundaciones (Balvanera, 2012). 
La superficie agrícola tuvo un incremento del $3.5 \%$ mientras que el uso pecuario presentó un leve descenso porcentual del 1.5\% (Cuadro 2). El crecimiento del uso agrícola ocurrió sobre la superficie pecuaria ( 370 ha) y en menor grado sobre humedales y vegetación arbórea (240ha); sin embargo, 497 ha de uso agrícola fueron desplazadas por el uso urbano (Figura 4). El incremento del área agrícola en el CPS concuerda con el auge agropecuario en Tabasco que inició en los años sesenta, con el impulso a la ganadería extensiva para abastecer al mercado nacional a través del Plan Chontalpa (1965-1976) y el Programa de Desarrollo Rural Integrado para el Trópico Húmedo (Flores-Santiago, 1987; Capdepont-Ballina y Marín-Olán, 2014).

En los años noventa, las políticas de apoyo al campo influyeron en la dinámica de uso del suelo en Tabasco (Murillo y Martínez, 2010). El Programa de apoyos directos al campo, PROCAMPO, propició la transformación de zonas de humedales y pastizales a superficies agrícolas, acelerando los procesos de cambio de uso del suelo (Klepeis y Vance, 2003; Schmook y Vance, 2009; ZarazúaEscobar et al., 2011). Además, en el estado de Tabasco, se impulsaron obras de drenaje para disminuir las inundaciones en zonas aledañas a humedales y fomentar la agricultura. En el CPS, a finales de los 80's, se ejecutaron obras de drenaje por el Gobierno del Estado y PEMEX, en respuesta a ejidatarios que reclamaban retención de agua por efecto de la infraestructura petrolera y las terracerías en el flujo del agua, lo que incrementó los usos agropecuarios (Zavala-Cruz et al., 2003; Zavala-Cruz y Castillo-Acosta, 2007).

En cuanto al uso pecuario, creció sobre 480 ha de coberturas de humedales y sobre 216 ha de vegetación arbórea, y perdió 305 ha por expansión de los usos agrícola, infraestructura petrolera y urbano (Figura 4). El aumento del uso pecuario en el CPS es congruente con el incremento de la superficie con pastizales para la ganadería en el estado de Tabasco, por efecto de programas de apoyo como el mejoramiento de praderas con especies de gramíneas más nutritivas en el periodo 1965-2019 (Zavala-Cruz y Castillo-Acosta, 2007), la implementación de créditos a productores para al establecimiento de pastizales y mejoramiento de instalaciones para ganado bovino, mediante el Programa de Producción Pecuaria Sustentable y Ordenamiento Ganadero y Apícola (PROGAN), a finales del siglo XX y la ejecución del Programa de Apoyos Directos al Campo (PROCAMPO) (Kolb et al., 2013).

El uso urbano en el CPS se expandió 100\% desde 1965 a la fecha (Cuadro 2), esto se debió al fenómeno de periurbanización de Villahermosa sobre áreas agrícolas y pecuarias (Palomeque-De la Cruz et al., 2017a). Dicho campo petrolero presenta características favorables para la urbanización, como: a) la posición geográfica, al ser punto de enlace entre las ciudades de Villahermosa (a $7 \mathrm{~km}$ ), Cárdenas, Cunduacán y Reforma y dos petroquímicas, a través de la carretera 180 que facilita la conectividad; b) suelos Fluvisoles excentos de inundaciones fluviales, al ubicarse sobre geoformas de diques naturales del río Carrizal con la topografía más elevada en la planicie; y c) la protección del área con bordos contra inundaciones (Zavala-Cruz et al., 2003; Zavala-Cruz et al., 2016).

Sin embargo, el cambio a uso urbano induce degradación de los Fluvisoles por sellamiento, no obstante que son los más fértiles y aptos para la producción de alimentos (Palma-López et al., 2017), al pasar de usos con vegetación natural o cultivada, a usos de viviendas, oficinas, vialidades e industria petrolera, que reducen la aptitud agrícola y la capacidad de infiltración de agua hacia el manto acuífero (Franco-Idarraga, 2010; García-Rodríguez y Pérez-González, 2011). La infraestructura petrolera del CPS incrementó 95.9\% (Cuadro 2) y creció sobre 104 ha 
de humedales y 106 ha de superficie pecuaria (Figura 4). El impulso de esta industria coincidió con el boom petrolero en Tabasco, basado en la explotación de nuevos yacimientos de hidrocarburos en el sureste de México entre 1973 y 1980, lo que produjo un cambio radical en la economía de Villahermosa y aceleró la deforestación en el estado (Allub y Michel, 1979; Sánchez-Munguía, 2005).

El CPS y la mayoría de los campos petroleros en Tabasco, se ubicaron en planicies de inundación, y la construcción de carreteras a través de los humedales para acceder a los pozos petroleros, modificaron los patrones de circulación natural del agua, ciclos biogeoquímicos y la biodiversidad de la fauna acuática, al carecer de pasos de agua eficientes (Zavala-Cruz et al., 2003; CapdepontBallina y Marin-Olan, 2014; Sánchez et al., 2015). Además, se generó contaminación de agua y suelo por derrames de hidrocarburos y descarga de desechos tóxicos (Gutiérrez-Castorena y Zavala-Cruz, 2001; Zavala-Cruz et al., 2003; Olguín et al., 2007).

El impacto ambiental en el CPS también se evidencia con la detección cartográfica y cuantitativa de las transiciones más sobresalientes durante el periodo 1965-2019, donde las actividades agrícolas y pecuarias en conjunto tuvieron mayor influencia (Figura 5). En primer lugar, se detectó el remplazo de 807 ha de superficie pecuaria por áreas agrícolas, seguido de la pérdida de 747.8 ha de humedales que se transformaron en uso pecuario (Figura 6). Otras transiciones que contribuyeron al deterioro ambiental fueron el crecimiento de 499.6 ha de la zona urbana sobre el uso agrícola y la deforestación de 400 ha de la vegetación arbórea para el establecimiento del uso pecuario (Figura 6).

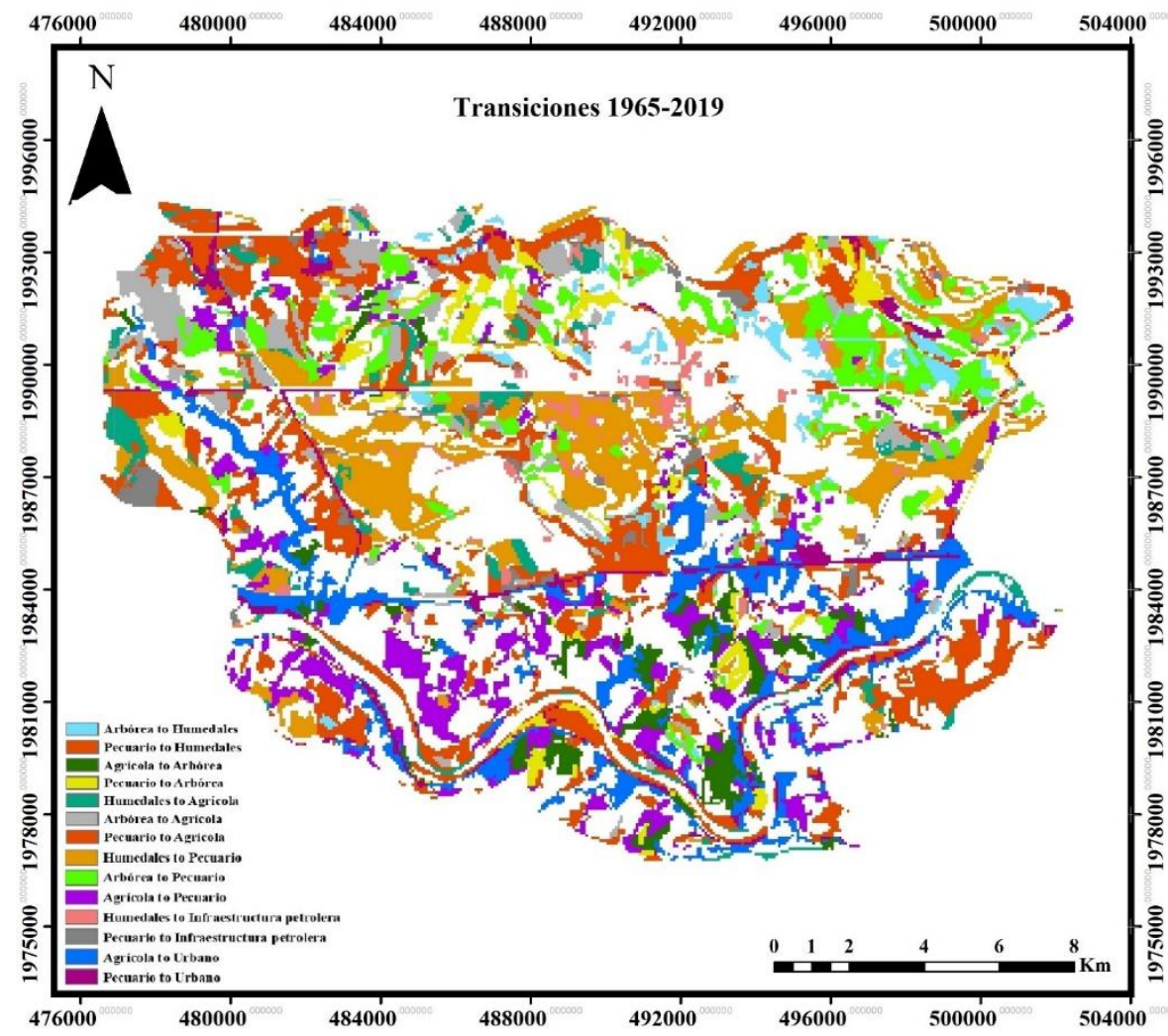

Figura 5. Mapa de transiciones de uso del suelo y coberturas en el campo petrolero Samaria, Tabasco (1965-2019). 


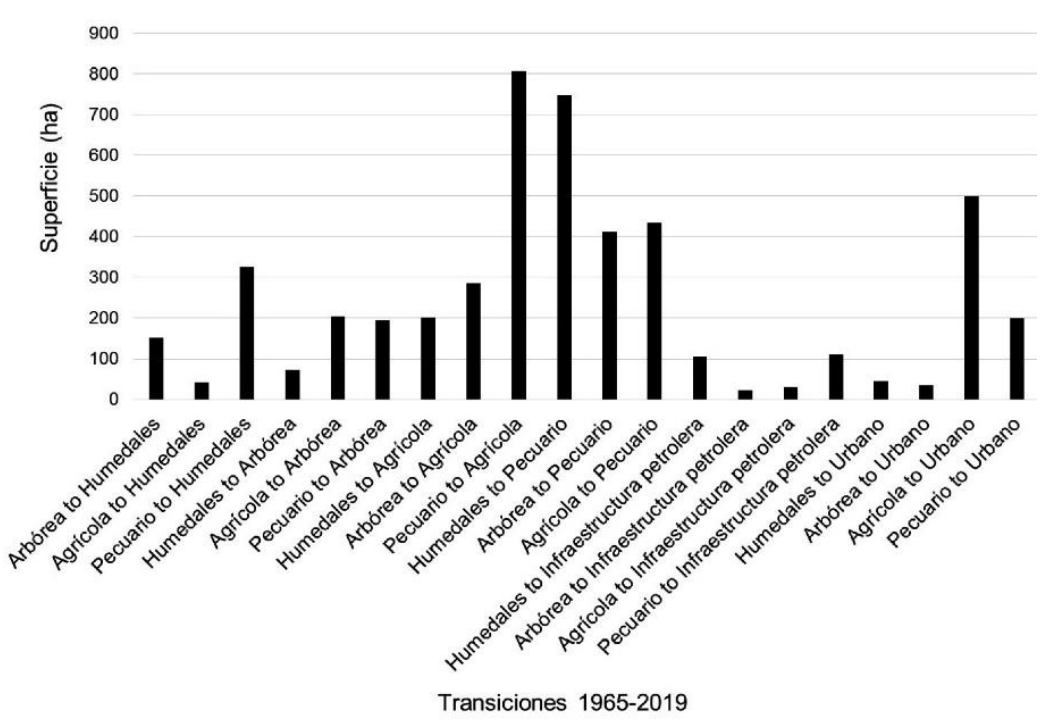

Figura 6. Grafica de transiciones de uso del suelo y coberturas en el campo petrolero Samaria, Tabasco (1965-2019).

El análisis de cambio de uso del suelo durante 1965-2019 indica que, de continuar la tendencia de perdida de humedales y vegetación en las próximas décadas por efecto del uso agropecuario y urbano, más los posibles impactos asociados a la reactivación de la industria petrolera en Tabasco, en campos petroleros nuevos y la nueva refinería en Dos Bocas, podrían desaparecer los últimos relictos de estos ecosistemas, magnificando la pérdida del hábitat de flora y fauna silvestre, la degradación de suelos agrícolas, la pérdida de espacios para recreación humana, y el deterioro de los servicios ambientales.

Las tierras del CPS son ejemplo del proceso de ganaderización y junto con la industria petrolera han provocado degradación de la vegetación natural y humedales, al no tomar en cuenta la capacidad de uso y potencial agroecológico de las tierras; por lo que es recomendable frenar el avance desordenado de estos usos. Las actividades de la industria petrolera y la construcción de carreteras deben ser planificadas con base en estudios de impacto ambiental y de ordenamiento ecológico, y priorizar el uso de las tierras para la producción agrícola y forestal (Zavala-Cruz y Castillo-Acosta, 2007).

Este estudio demuestra que los modelos geomáticos de cambio de uso del suelo para la evaluación de la degradación de los ecosistemas por cambio de uso del suelo son precisos en la estimación de la distribución del cambio de coberturas naturales y usos artificiales, e identifica las coberturas que presentan los mayores impactos ambientales (Velázquez et al., 2002). Es pertinente combinar modelos geomáticos para el estudio del cambio de uso del suelo, y simular escenarios futuros y las variables impulsoras del potencial de transición (Palomeque-De la Cruz et al., 2017b).

\section{Conclusiones}

El estudio de cambio de uso del suelo en el Campo Petrolero Samaria (CPS), Tabasco, evidencia que en el periodo 1964-2019, el establecimiento de pastizales para la ganadería dominó el territorio, seguidos de cultivos agrícolas e infraestructura petrolera, en conjunto provocaron degradación de los ecosistemas de humedales y vegetación arbórea. Actualmente los usos agropecuarios tienden a 
disminuir por efecto de la urbanización impulsada desde la ciudad de Villahermosa. La modelación utilizando land change modeler, revela que es una herramienta adecuada para evaluar cambios de uso del suelo y estimar la distribución del cambio de coberturas naturales y usos artificiales, en zonas con uso agropecuario, urbano e industria petrolera.

\section{Literatura citada}

Allub, L. y Michel, M. A. 1979. La formación socioeconómica de Tabasco y el petróleo. Investigación Económica. 38(148/149):327-355. http://www.jstor.org/stable/42777039.

Balvanera, P. 2012. Los servicios ecosistémicos que ofrecen los bosques tropicales. Ecosistemas. 21(1-2):136-147.

Bazant, J. 2010. Expansión urbana incontrolada y paradigmas de la planeación urbana. Espacio Abierto. 19(3):475-503.

Brena, J.; Castillo, C. y Wagner, A. 2016. Metodología para la delimitación y caracterización de humedales en escalas 1:50,000 y 1:20,000. Tecnología y Ciencias del Agua. 7(2):85-98.

Camacho-Olmedo, M. T.; Molero-Melgarejo, E. y Paegelow, M. 2010. Modelos geomáticos aplicados a la simulación de cambios de usos del suelo. Evaluación del potencial de cambio. In: Ojeda, J.; Pita, M. F. y Vallejo, I. (Ed.). Tecnologías de la información geográfica: la información geográfica al servicio de los ciudadanos. Secretariado de publicaciones de la Universidad de Sevilla. Sevilla, España. 658-678 pp.

Capdepont-Ballina, J. L. y Marín-Olán, P. 2014. La economía de Tabasco y su impacto en el crecimiento urbano de la ciudad de Villahermosa (1960-2010). LiminaR. 12(1):144-160. https://doi.org/10.29043/liminar.v12i1.330.

Challenger, A. y Dirzo, R. 2009. Tendencias de cambio de la biodiversidad. In: capital natural de México vol. II: Estado de conservación y tendencias de cambio. Comisión Nacional de la Biodiversidad (CONABIO). México, DF. 37-73 pp.

Davidson, E. A.; de Araújo, A. C.; Artaxo, P.; Balch, J. K.; Brown, I. F.; Bustamante, M. M. C.; Coe, M. T. C.; DeFries, R. S.; Keller, M.; Longo, M.; Munger, J. W.; Schroeder, W.; Soares-Filho, B. S.; Souza Jr., C. M. and Wofsy, S. C. 2012. The Amazon Basin in transition. Nature. 481:321-328. http://www.nature.com/doifinder/10.1038/nature10717.

Eastman, J. R. 2012. IDRISI Selva GIS and image processing software version 17.0. Clark Labs. Massachusetts, USA. 321 p. https://clarklabs.org/wp-content/uploads/2016/10/IDRISISelva-Spanish-Manual.pdf.

FAO. 2015. Organización de las Naciones Unidas para la Alimentación y la Agricultura. Evaluación de los recursos forestales mundiales 2015 compendio de datos. Roma, Italia. 245 p. http://www.fao.org/3/a-i4808s.pdf.

Flores-Santiago, A. 1987. La modernización de la agricultura en el trópico húmedo mexicano: veinte años de experiencia en la Chontalpa, Tabasco. Rev. Geogr. Agríc. 13-14(2):105-114.

Franco-Idarraga, F. L. 2010. Respuestas y propuestas ante el riesgo de inundación de las ciudades colombianas. Rev. Ing. 31:97-108. https://doi:10.16924/riua.v0i31.216.

García-Rodríguez, M. y Pérez-González, M. E. 2011. Sellado de fluvisoles en la comunidad de Madrid. Análisis a partir de imágenes Landsat. Anales de Geografía de la Universidad Complutense. 31(2):125-137. https://doi.org/10.5209/rev_AGUC.2011.v31.n2.37021.

Guerra-Martínez, V. y Ochoa-Gaona, S. 2006. Evaluación espacio-temporal de la vegetación y uso del suelo en la Reserva de la Biosfera Pantanos de Centla, Tabasco (1990-2000). Investigaciones Geogr. 59:7-25. http://www.investigacionesgeograficas.unam.mx/ index.php/rig/article/viewFile/30018/27897. 
Gutiérrez-Castorena, M. C. y Zavala-Cruz, J. 2001. Rasgos hidromórficos de suelos tropicales contaminados con hidrocarburos. Terrra Latinoam. 20(2):101-111.

Hettiarachchi, M.; Morrison, T. H.; Wickramsinghe, D.; Mapa, R.; De Alwis, A. and McAlpine, C. A. 2014. The eco-social transformation of urban wetlands: a case study of Colombo, Sri Lanka. Landscape and Urban Planning. 132:55-68. https://doi.org/10.1016/ j.landurbplan.2014.08.006.

Jiménez-Moreno, M. J.; González-Guillen, M. D. J. Escalona-Maurice, M.; Valdez-Lazalde, J. R. y Aguirre-Salado, C. A. 2011. Comparación de métodos espaciales para detectar cambios en el uso del suelo urbano. Rev. Chapingo Ser. Cienc. Forest. Amb. 17(3):389-406. doi: 10.5154./r.rchscfa.2010.04.020.

Klepeis, P. and Vance, C. 2003. Neoliberal policy and deforestation in southeastern Mexico: an assessment of the Procampo program. Econ. Geography. 79(3):221-240. https://www.jstor.org/stable/30032931.

Kolb, M.; Mas, J. F. and Galicia, L. 2013. Evaluating drivers of land-use change and transition potential models in a complex landscape in Southern Mexico. Int. J. Geogr Inf. Sci. 27(9):1804-1827. doi:10.1080/13658816.2013.770517.

Lezama, J. L. 1987. Migración y petróleo en Tabasco. Estudios demográficos y urbanos. 2(2):231256. https:// doi.org/10.24201/edu.v2i2.627.

Murillo, L. D. y Martínez, R. J. 2010. Comunicación para el desarrollo en México: reflexiones sobre una experiencia en el trópico húmedo. Estudios sobre las Culturas Contemporáneas. 16(31):201-225. https://www.redalyc.org/pdf/316/31613952008.pdf.

Olguín, E. J.; Hernández, M. E. y Sánchez-Galván, G. 2007. Contaminación de manglares por hidrocarburos y estrategias de biorremediación, fitorremediación y restauración. Rev. Int. Cont. Amb. 23(3):139-154. https://www.redalyc.org/pdf/370/37023304.pdf.

Ortiz-Solorio, C. A.; Gutiérrez-Castorena M. del C.; Sánchez-Guzmán, P. y Gutiérrez-Castorena, E. V. 2011. Cartografía de la degradación de suelos en la República Mexicana: evolución y perspectivas. In: Krasilnikov, P.; Jiménez-Nava, F. J.; Reyna-Trujillo, T. y GarcíaCalderón, N. E. (Ed.). Geografía de suelos de México. Facultad de Ciencias-Universidad Nacional Autónoma de México (UNAM). México, D F. 173-210 pp.

Paegelow, M.; Camacho, M. y Menor, J. 2003. Cadenas de Markov, evaluación multicriterio y evaluación multiobjetivo para la modelización prospectiva del paisaje. GeoFocus. 3:22-44. http://www.geofocus.org/index.php/geofocus/article/view/21.

Palacio-Prieto, J. L; Sánchez-Salazar, M. T.; Casado, I. J. M.; Propin, F. E.; Delgado, C. J.; Velázquez, M. A.; Chías, B. L.; Ortiz, A. M. I.; González, S. J.; Negrete, F. G.; Gabriel, M. J. y Márquez, H. R. 2004. Indicadores para la caracterización y el ordenamiento territorial. Secretaría de Medio Ambiente y Recursos Naturales (SEMARNAT)-Instituto Federal Electoral (INE)-Universidad Nacional Autónoma de México (UNAM). Instituto de Geografía y SEDESOL. México, DF. 161 p.

Palma-López, D. J.; Jiménez-Ramírez, R.; Zavala-Cruz, J.; Bautista-Zúñiga, F.; Gavi-Reyes, F. y Palma-Cancino, D. Y. 2017. Actualización de la clasificación de suelos de Tabasco. Agroproductividad. 10(12):29-35.

Palomeque-De la Cruz, M. A.; Galindo-Alcántara, A.; Sánchez, A. J. y Escalona-Maurice, M. J. 2017a. Pérdida de humedales y vegetación por urbanización en la cuenca del río Grijalva, México. Investigaciones Geográficas. 68:151-162. https://www.redalyc.org/ pdf/176/17653923009.pdf.

Palomeque-De la Cruz, M. A.; Galindo-Alcántara, A.; Pérez-Sánchez, E.; Sánchez, A. D. J. y Escalona-Maurice, M. J. 2017b. Modelos geomáticos con base en transición para el análisis espacial en Villahermosa, Tabasco. Rev. Mex. Cienc. Agríc. 8(2):253-267. http://www.scielo.org.mx/pdf/remexca/v8n2/2007-0934-remexca-8-02-253-en.pdf. 
PEMEX. 2016. Petróleos Mexicanos. Anuario estadístico 2016. Ciudad de México. 122 p. https://www.pemex.com/ri/Publicaciones/Anuario\%20Estadistico\%20Archivos/anuarioestadistico-2016.pdf.

Pineda-Jaimes, N. B.; Bosque-Sendra, J.; Gómez-Delgado, M. y Plata-Rocha, W. 2009. Análisis de cambio del uso del suelo en el Estado de México mediante sistemas de información geográfica y técnicas de regresión multivariantes. Una aproximación a los procesos de deforestación. Investigaciones Geogr. 69:33-52. https://www.redalyc.org/pdf/569/ 56912295004.pdf.

Pontius Jr. R. G.; Huffaker, D. and Denman, K. 2004. Useful techniques of validation for spatially explicit land-change models. Ecological Modelling. 179:445-461. https://doi.org/10.1016/ j.ecolmodel.2004.05.010.

Ramos-Reyes, R.; Sánchez-Hernández, R. y Gama-Campillo, L. M. 2016. Análisis de cambios de uso del suelo en el municipio costero de Comalcalco, Tabasco, México. Ecosistemas y Recursos Agropecuarios. 3(8):151-160.

Rosete-Vergés, F. A.; Pérez-Damián, J. L.; Villalobos-Delgado, M.; Navarro-Salas, E. N.; SalinasChávez, E. y Remond-Noa, R. 2014. El avance de la deforestación en México 1976-2007. Madera y Bosques. 20(1):21-35. https://doi.org/10.21829/myb.2014.201173.

Sánchez, A.J.; Salcedo, M.A.; Florido, R.; Mendoza, J.D.; Ruiz-Carrera, V. y Álvarez-Pliego, N. 2015. Ciclos de inundación y conservación de servicios ambientales en la cuenca baja de los ríos Grijalva-Usumacinta. Contactos. 97:5-14. http://www2.izt.uam.mx/newpage/ contactos/revista/97/pdfs/inundacion.pdf.

Sánchez-Munguía, A. 2005. Uso del suelo agropecuario y desforestación en Tabasco 1950-2000. Universidad Juárez Autónoma de Tabasco. Villahermosa, Tabasco, México. 123 p.

Schmook, B. and Vance, C. 2009. Agricultural policy, market barriers, and deforestation: the case of Mexico's southern Yucatán. World Development. 37(5):1015-1025.

Torres-Vera, M. A.; Prol-Ledesma, R. M. and García-López, D. 2009. Three decades of land use variations in Mexico City. Int. J. Remote Sens. 30(1):117-138. https://doi.org/10.1080 /01431160802261163.

Trucíos-Caciano, R.; Rivera-González, M.; Delgado-Ramírez, G.; Estrada-Ávalos, J. y CeranoParedes, J. 2013. Análisis sobre cambio de uso de suelo en dos escalas de trabajo. Terra Latinoam. 31(4):339-346.

Tubiello, F. N.; Salvatore, M.; Ferrara, A. F.; House, J.; Federici, S.; Rossi, S.; Biancalani, R.; Condor, G. R. D.; Jacobs, H.; Flammini, A.; Prosperi, P.; Cardenas-Galindo, P.; Schmidhuber, J.; Sanz, S. M. J.; Srivastava, N. and Smith, P. 2015. The contribution of agriculture, forestry and other land use activities to global warming, 1990-2012. Global Change Biol. 21(7):2655-2660.

Tudela, F. 1992. La modernización forzada del trópico: El caso de Tabasco. Proyecto integrado del golfo. $2^{\mathrm{a}}$ (Ed.). El Colegio de México, CINVESTAV, INFIAS y UNRISD. México, DF. $474 \mathrm{p}$.

Velázquez, A.; Mas, J. F.; Gallegos, J. R. D.; Mayorga-Saucedo, R.; Alcántara, P. C.; Castro, R. y Palacio, J. L. 2002. Patrones y tasas de cambio de uso del suelo en México. Gaceta Ecológica. 62:21-37. https://www.redalyc.org/pdf/539/53906202.pdf.

Vitousek, P. M.; Mooney, H. A.; Lubchenco, J. and Melillo, J. M. 1997. Human domination of Earth's ecosystems. Science. 277(5325):494-499. Doi: 10.1126/science.277.5325.494.

Zarazúa-Escobar, J. A.; Almaguer-Vargas, G. y Ocampo-Ledesma, J. G. 2011. El programa de apoyos directos al campo (PROCAMPO) y su impacto sobre la gestión del conocimiento productivo y comercial de la agricultura del Estado de México. Agric. Soc. Des. 8(1):89-105. 
Zavala-Cruz, J.; Gutiérrez-Castorena, M. del C. y Palma-López, D. J. 2003. Impacto ambiental en las tierras del campo petrolero Samaria, Tabasco. Colegio de postgraduados. CCYTETCONACYT. Villahermosa, Tabasco. $131 \mathrm{p}$.

Zavala-Cruz, J. y Castillo-Acosta, O. 2007. Cambio de uso de la tierra en el estado de Tabasco. In: Palma D. J. y Triano, A. (Ed.). Plan de uso sustentable de los suelos del estado de Tabasco (vol, II). Colegio de Posgraduados, ISPROTAB. Villahermosa, México. 38-56 pp.

Zavala-Cruz, J.; Morales-Garduza, M. A.; Vargas-Villamil, L. M.; Palma-López, D. J. y OrtizSolorio, C. A. 2016. Capacidad de uso del suelo urbano en planicies fluviales costeras: el caso de Villahermosa, Tabasco, México. Interciencia. 41(5):296-304.

Zepeda-Gómez, C.; Nemiga, X. A.; Lot-Helgueras, A. y Madrigal-Uribe, D. 2012. Análisis del cambio del uso del suelo en las Ciénegas de Lerma (1973-2008) y su impacto en la vegetación acuática. Investigaciones Geográficas. 78:48-61. https://www.redalyc.org/ articulo.oa?id=569/56924410005. 